\title{
Comparison of English Language Rhythm and Kalhori Kurdish Language Rhythm
}

\author{
Nafiseh Taghva (Corresponding author) \\ Department of Linguistics and Foreign Languages, Payamenoor University,19395- 4679,Tehran,I.R.Iran \\ E-mail: n.taghva@yahoo.com \\ Vahideh Abolhasani Zadeh \\ Shahid Bahonar, University of Kerman, Kerman, Iran \\ E-mail: abolhasanizadeh@yahoo.com
}

Doi:10.7575/aiac.alls.v.7n.2p.226

Received: 20/12/2015

URL: http://dx.doi.org/10.7575/aiac.alls.v.7n.2p.226

Accepted: 03/02/2016

\begin{abstract}
Investigating on quantitative features of languages rhythm is a recent issue that attracts the attention of linguists. Interval-based method is a method of studying the rhythmic quantitative features of languages. This method use Pairwise Variability Index (PVI) to consider the variability of vocalic duration and inter-vocalic duration of sentences which leads to classification of languages rhythm into stress-timed languages and syllable-timed ones. This study aims to consider the rhythm of British English and Kalhori Kurdish, which is spoken in some part of west of Iran, based on interval-based method. In order to reach this aim the duration variability of vocalic interval and inter-vocalic interval of English and Kalhori Kurdish are measured by PVI. Afterward the outcomes of this study were compared to the existed results of other languages. The results of this research demonstrated that the rhythmic quantitative features of these two languages are placed among stress-timed languages.
\end{abstract}

Keywords: Rhythm, interval-based method, syllable-timed, stress-timed

\section{Introduction}

Heartbeats, waves of the sea and birds songs are natural phenomenon that are benefited from rhythm. The word rhythm

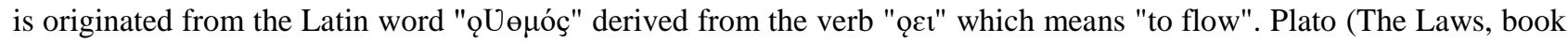
II: 93) defined rhythm as order in movement. Languages are among natural phenomenon which includes rhythm. Patel (2010) stated: "Rhythm is a systematic patterning of sound in terms of timing, accent and grouping. Both speech and music are characterized by systematic, temporal, accentual and phrasal patterning."

In the present paper the acoustic-phonetic basis of English rhythm and Kalhori Kurdish rhythm are investigated. Kurdish language is a branch of Indo-European languages which is spoken in vast area of Iran, Iraq, Turkey and Saria and even some parts of Middle East. Kalhori Kurdish is a subcategory of Kurdi in southern area of Kurdish language that is spoken in west part of Iran (Gudarzi, 2003).

The first attempts of studying rhythm began by the hypothesis of relation between rhythm and duration. Lloyd James (1940) had studied rhythm of some languages by comparing their prosodic differences. He said that the same elements repeated at the same regular intervals of time. Pike (1945) named these intervals as 'syllable-timed' and 'stress-timed'. Abercrombie (1967) presented the theory of Isochrony and claimed that inter-stress intervals were the same in stresstimed languages but in syllable-timed ones inter-syllabic intervals were the same. Subsequent research added another type to this classification as Mora timed languages in which morae are said to be near-equal in duration (Ladefoged, 1975). However, later work (Bolinger, 1965; Wenk, Wiolland, 1982; Manrique \& Signorini, 1983) presented counter arguments against the theory of Isochrones. Hense the Theory of Isocrony was violated.

On the other hand, Dauer (1983) demonstrated some phonetic and phonological differences between stress-timed languages and syllable-timed languages. Not only he stated that syllables variety were greater in stress-timed languages than syllable-timed ones, but also he showed that unstressed syllables had vowel reduction in stress-timed languages. Besides, Dauer (1987) hypothesized a continuum in which stress-timed languages were placed on one end and syllabletimed languages on the other end. This idea was supported by Nespor (1990), Bertoncini (1989), and Dasher and Bolinger (1982).

Ramus et al. (1999) presented three factors of rhythm, the proportion of vocalic intervals in the sentence (\%V), the standard deviation of vocalic intervals within a sentence $(\Delta \mathrm{V})$ and the standard deviation of consonantal intervals within a sentence $(\Delta \mathrm{C})$. Their findings suggested that as more syllable types show more variability in the number of consonant and more duration variability of syllable that cause more $\Delta \mathrm{C}$ and less $\% \mathrm{~V}$, there is a negative relationship between $\Delta \mathrm{C}$ and $\% \mathrm{~V}$. 
After Ramus researches some other attempts have done to measure the duration of speech. One of them leaded to the introduction of Pairwise variability Index, PVI (Grabe \& Low, 2002). Ladefoged and Johnson (2011: 252) accepted four procedures to calculate PVI:

1) Identification of the interval which should be measured (vocalic interval or intervocalic interval)

2) Calculation of duration difference between each pair of adjacent intervals in the utterance

3) Division of each result by the mean duration of each pair

4) Setting up the average ratio of each pair

Grabe and Low (2002) confirmed that vocalic nPVI, normalized Pairwise Variability Index, and intervocalic rPVI , raw Pairwise Variability Index, is a better criterion to determine the rhythmic class of languages than vocalic nPVI alone. Their findings indicated that vowel durational variability is greater in stress-timed languages such as German and English than in syllable-timed languages such as French and Spanish. Therefore the logic of PVI is that languages can be classified in accordance to the vocalic durational intervals, intervocalic durational intervals and the contrast of these successive durations (Ramus et al., 1999 and Grabe \& Low, 2002).

$$
n P V I=\frac{100}{m-1} \times \sum_{k=1}^{m-1}\left|\frac{d_{k}-d_{k+1}}{d_{k}+d_{k+1}}-\right|
$$

Where $m$ is the number of vocalic intervals in an utterance and $\mathrm{d}_{\mathrm{k}}$ is the duration of the kth interval.

$$
r P V I=\frac{100}{m-1} \times \sum_{k=1}^{m-1}\left|d_{k}-d_{k+1}\right|
$$

Where $m$ is the number of inter- vocalic intervals in an utterance and $\mathrm{d}_{\mathrm{k}}$ is the duration of the kth interval.

Table 1. PVI of different languages (derived from Grabe \& Low.2002)

\begin{tabular}{lll}
\hline Language & nPVI & rPVI \\
\hline Thai & 65.8 & 56.5 \\
\hline Dutch & 65.5 & 57.4 \\
\hline German & 59.7 & 55.3 \\
\hline British English & 57.2 & 64.1 \\
\hline Tamil & 55.8 & 70.2 \\
\hline Malay & 53.6 & 66.3 \\
\hline Greek & 48.7 & 59.6 \\
\hline Welsh & 48.2 & 54.7 \\
\hline Rumanian & 46.9 & 47.6 \\
\hline Polish & 46.6 & 79.1 \\
\hline Catalan & 44.6 & 67.8 \\
\hline French & 43.5 & 50.4 \\
\hline Japanese & 40.9 & 62.5 \\
\hline Luxembourg & 37.7 & 55.4 \\
\hline Spanish & 29.7 & 55.7 \\
\hline Mandarin & 27 & 52 \\
\hline
\end{tabular}

PVI have been studied by many linguists during the last decades which can approve the efficiency of this measurement in analyzing the acoustic rhythmic features of languages (Dellwo, 2006; White \& Mattys, 2007; Nolan \& Asu, 2009; Patel, 2010). Tilsen and Arvaniti (2013) called this method as "Interval-based" method.

Although many works have been done on phonetic and phenology of Kurdish language (such as Karimi Dustan, 2003 and Fatahi, 2013), none of them have considered the quantitative rhythmic features of this language.

\section{Method}

In order to investigate the rhythmic features of English and Kurdish, and in order to compare them with other languages, this study use the interval-based method to measure specific aspects of rhythmic dimensions. Hence, this 
research was used Grabe andLow's (2002) method by calculating vocalic nPVI and intervocalic rPVI of these two languages.

Therefore, 4 native speakers of English read 40 simple declarative English sentences in a silent room. These sentences were analyzed using the software Praat. Then text grids will be made for each sentence in a way that the boundaries of each vowel and consonant were determined. Afterward, the durational variability of vowels and consonants were measured by nPVI and rPVI. Subsequently, all these procedures were done for 40 declarative Kalhori Kurdish sentences which were read by 4 native speakers of this language. An example of a simple declarative English sentence TextGrid is presented in Figure 1 and an example of a simple declarative Kurdish sentence TextGrid is presented in Figure 2 .

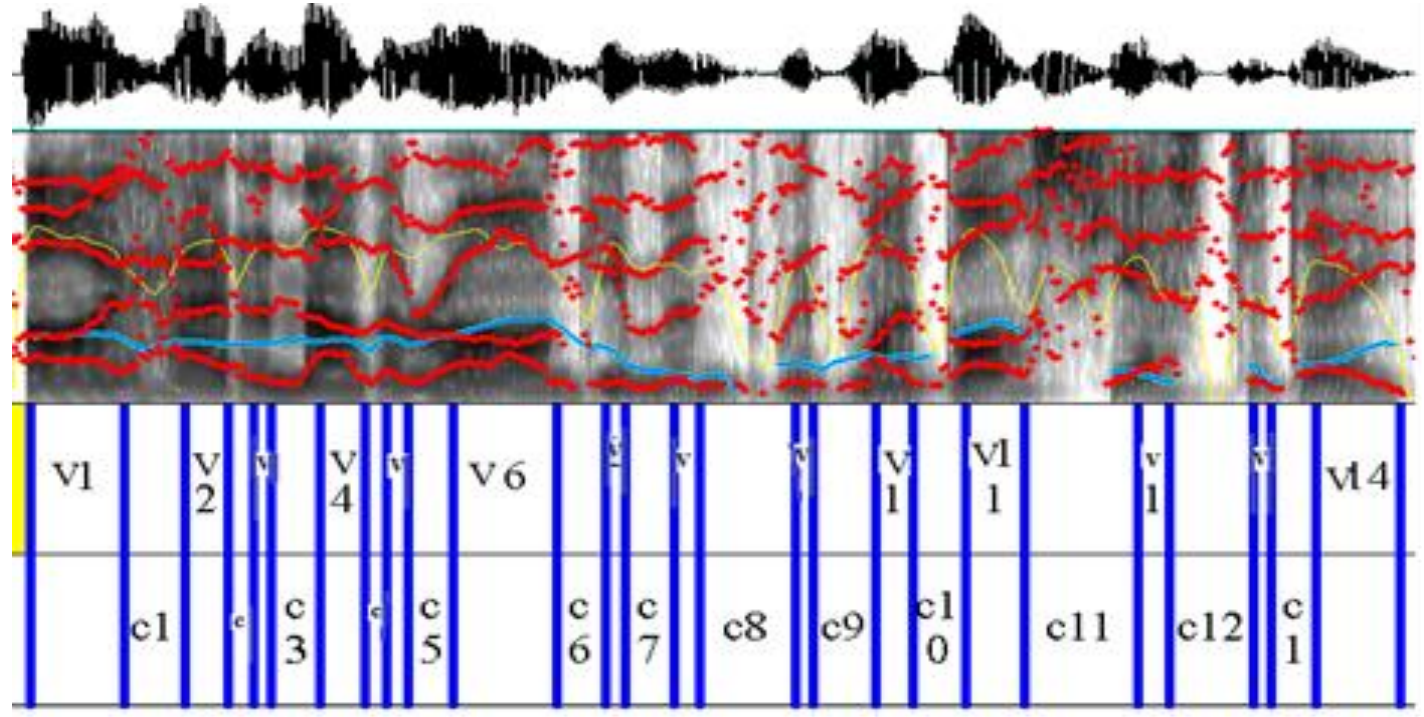

Figure 1. An example of a Textgrid for a simple declarative English sentence (I had another argument with my boss yesterday)

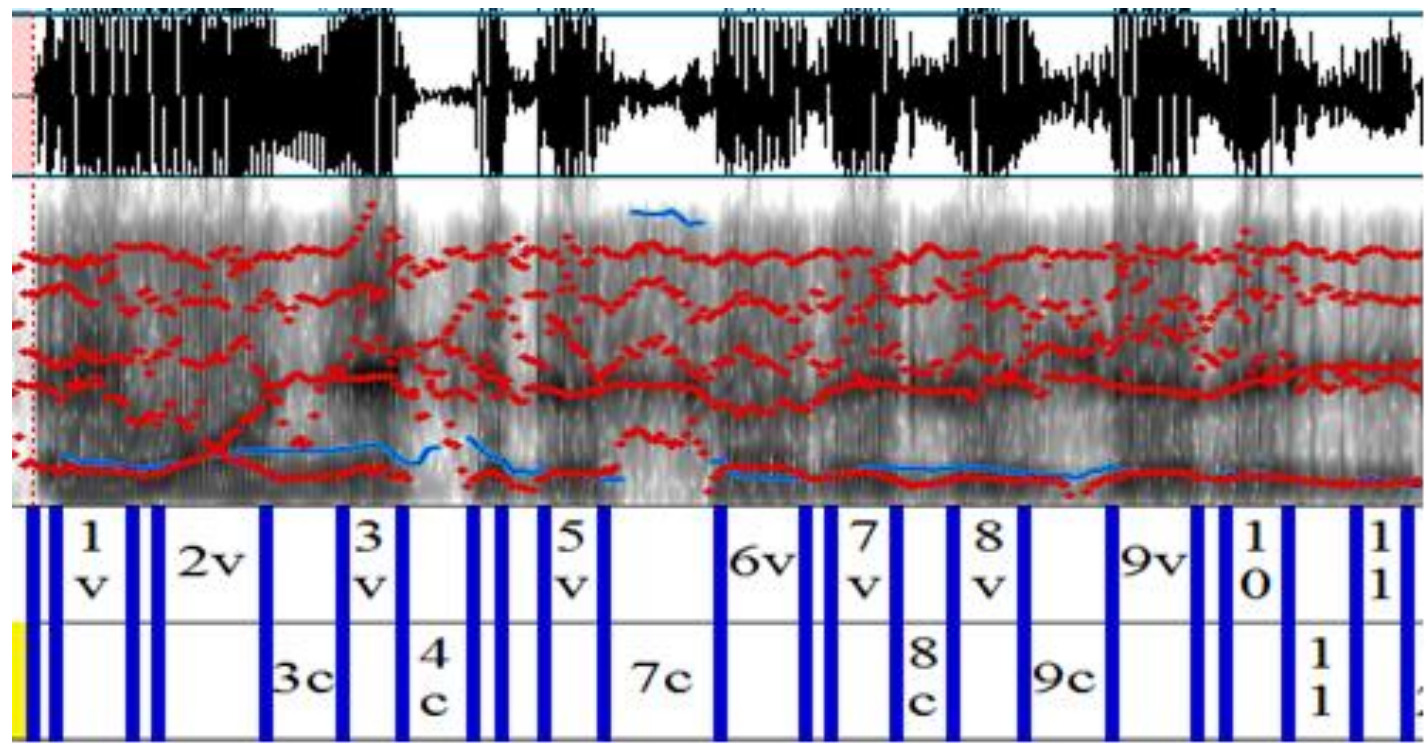

Figure 2. An example of a Textgrid for a simple declarative English sentence /eiwan jeki we sharjele ilame/. Ivan is one of the biggest city of Ilam

\section{Results}

The results of the study by calculating vocalic nPVI and inter-vocalic rPVI of 40 simple declarative English sentences (nPVI: 57.88 and rPVI: 67) demonstrated that British English is placed among stress-timed languages. Besides, vocalic nPVI and inter-vocalic rPVI of 40 simple declarative Kalhori Kurdish sentences (nPVI: 54 and rPVI: 52.88) put the the rhythmic features of this language among stress-timed languages, too. Figure 3 indicates the nPVI of these two languages and compares them to the existed nPVI of some other languages derived from Ladefoged \& Johnson, 2011:253). 


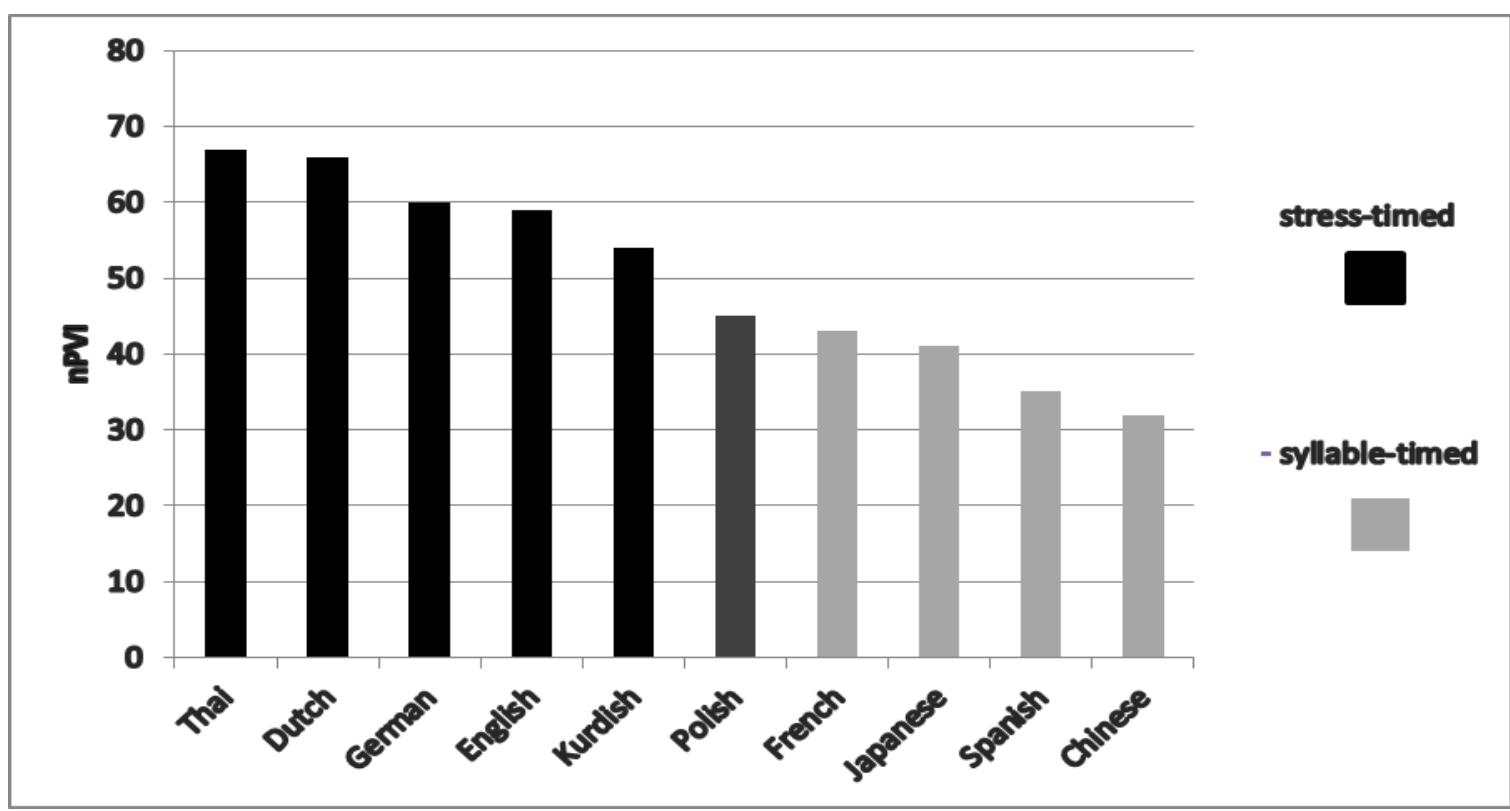

Figure 3. Comparison of English and Kurdish nPVI with other languages nPVI derived from Ladefoged \&Johnson, 2011:253)

As you can observe in Figure 4, though the nPVI of these two languages are not differ so much their rPVI are not the same.

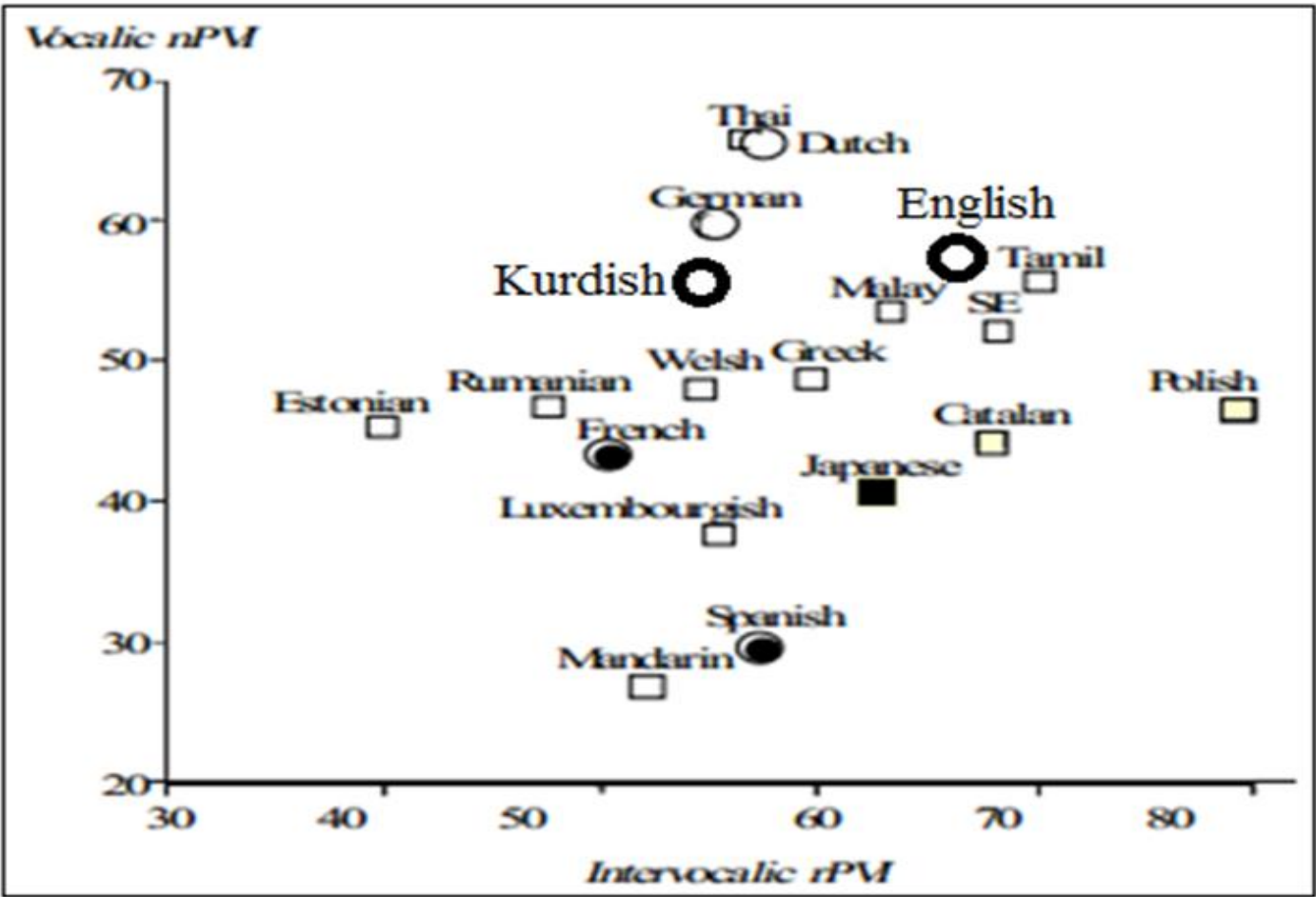

Figure 4. Comparison of English and Kurdish PVI with other languages PVI (derived from Grabe \& Low, 2002)

\section{Discussion \& Conclusion}

The speech rhythm study is a recent endeavor in linguistics in spite of long history study of rhythm in poetry (Patel, 2010:118). As language, within the human mind, perform interpretive operation by changing complex acoustic sequences into separate elements and organizing them into hierarchical structures to convey rich meanings, the study of speech rhythm can be beneficial for different scientists such as biologists, poets, composers, linguists and musicians.

This study is an attempt to identify the rhythmic class of English and Kalhori Kurdish. In order to reach this aim the rhythmic features of English and Kalhori Kurdish were measured (Table 2) and then they were compared to other languages existed rhythmic features. The results of this study show that the rhythm of English and Kurdish are placed in the stress-timed languages category. 
Table 2. Rhythmic features of English and Kalhori Kurdish

\begin{tabular}{l|ll}
\hline Rhythmic features & English & Kalhori Kurdish \\
\hline Vocalic nPVI & 57.88 & 54.22 \\
Inter-vocalic rPVI & 67 & 52.88 \\
\hline
\end{tabular}

Science Kalhori Kurdish is spoken in some west part of Iran and Iran is country with different languages, investigating on rhythmic features of these languages can be a good idea for further researches.

Recently, nPVI is used to compare language and music rhythm that have presented the trace of language rhythm of a culture on instrumental music rhythm of that culture (Patel, 2010: 130-179). Hence, studying the nPVI of Kurdish music can help the better understanding of this claim.

\section{Acknowledgements}

We acknowledge the native speakers of British English and Kalhori Kurdish who read the sentences of this study.

\section{References}

Abercrombie, D. (1967). Elements of General Phonetics. Chicago: Aldine.

Bertinetto, P. M. (1989). Reflections on the Dichotomy 'Stress' vs. 'Syllable Timing'. Rev. Phonet, Appl, 91-93, 99129.

Bolinger, D.L. (1965). Forms of English: Accent, Morpheme, Order. Cambridge, Massachusetts: Harvard University Press.

Dasher, R. \& Bolinger, D. (1982). On pre-accentual lengthening. Journal of the International Phonetic Association, 12, 58-69.

Dauer, R.M. (1983). Stress-timing and syllable-timing re-analysed. Journal of Phonetics 11, 51-62.

Dauer, R. M. (1987). Phonetic and Phonological Components of Language Rhythm. In Proceedings of the 11th International Congress of Phonetic Sciences. Tallinn, 447-449.

Dellwo, V. (2006). Rhythm and Speech Rate: A Variation Coefficient for DeltaC. In Language and LanguageProcessing: Proceedings of the $38^{\text {th }}$ Linguistics Colloquium. Piliscsaba 2003, edited by P. Karnowski and I.Szigeti (Peter Lang, Frankfurt am Main), 231-241.

Fatahi, M. (2013). Glides in Kalhori Kurdish. In proceedings of the $8^{\text {th }}$ Conference on Linguistics. Tehran: Allame Tabatabai University, 616-630.

Gudarzi, E. (2003). Kalhor tribe in Mashrutiat era. Kermanshah: Kermanshah, 32.

Grabe, E., \& Low, E. L. (2002). Durational Variability in Speech and the Rhythm Class Hypothesis. In C. Gussenhoven \& N. Warner, Laboratory phonology. Berlin: Mouton de Gruyter, 515-546.

Karimi Dustan, Q. (2003). Syllable structure in Kurdish.The journal of Humanities in Mashhad University, 35, 235-248.

Ladefoged, P. (1975). A Course in Phonetics. New York: Harcourt Brace Jovanovich.

Ladefoged, P., \& Johnson, K. A., (2011). Course in Phonetics ( $6^{\text {th }}$ ed.). Wadsworth: Boston, 252-253.

Lloyd James, A. (1940). Speech Signals in Telephony. London: Pitman and Sons, 16-27.

Manrique, A.M.B. \& Signorini, A. (1983). Segmental reduction in Spanish. Journal of Phonetics, 11, 117-128.

Nespor, M. (1990). On the Rhythm Parameter in Phonology. In I. M. Roca, Logical issues in language acquisition. Dordrecht: Foris, 157-175.

Nolan, F., \& Asu, E. L. (2009). The Pairwise Variability Index and Coexisting Rhythms in language. Phonetica, 66, 64-77.

Patel A, D. (2010). Music, Language, and the Brain. New York: Oxford University Press.

Pike, K. L. (1945). The Intonation of American English. Ann Arbor, MI: University of Michigan Press. Plato. The Laws. book II, 93.

Ramus, F., Nespor, M., and Mehler, J. (1999). Correlates of linguistic rhythm in the speech signal. Cognition, 75, 265292.

Tilsen, S., \& Arvaniti, A. (2013). Speech rhythm analysis with decomposition of the amplitude envelope: Characterizing Rhythmic Patterns within and across Languages. Acoustical Society of America.

Wenk, B. \& Wioland, F. (1982). Is French really syllable-timed? Journal of Phonetics, 10, 193-216.

White, L., \& Mattys, S. L. (2007). Calibrating Rhythm: First Language and Second Language Studies. J. Phonetics, 35, $501-522$. 